\title{
MOTIVACIÓN A LA LECTURA Y \\ PREFERENCIA DE LOS GÉNEROS \\ LITERARIOS SEGÚN EL SEXO \\ DE ESTUDIANTES
}

\section{MOTIVATION TO READING AND PREFERENCE OF LITERARY GENRES RELATED TO STUDENTS' GENDER}

Luzmila Flores Correa

Gabriela Verónica Alcalá Adrianzén

Mgtr. en Educación con Mención en Teoría y prácticas educativas. Docente ordinaria en la sección académica de Pedagogía, Facultad de Ciencias de la Educación, Universidad de Piura.

luzmila.flores@udep.pe

Mgtr. en Educación con Mención en Psicopedagogía. Jefa de la sección académica de Educación Primaria, Facultad de Ciencias de la Educación, Universidad de Piura.

gabriela.alcala@udep.pe

\section{RESUMEN}

Para desarrollar el proceso lector se necesita de una disposición que permita al lector relacionarse mejor con el texto. Siendo la motivación un aspecto importante, podemos plantear las siguientes interrogantes: ¿quiénes están más motivados: los niños o las niñas?, ¿por qué?, ¿qué género literario prefieren leer, según su sexo? 
La presente investigación involucró a 400 niños de Inicial de 5 años, y $1^{\circ}$ y $2^{\circ}$ grado de Primaria de colegios de distinto tipo de gestión, a quienes se les aplicaron dos cuestionarios, uno sobre motivación a la lectura y otro sobre géneros literarios (su contacto y preferencia).

Los resultados evidenciaron las diferencias existentes entre niños y niñas (a nivel de motivación y preferencias sobre los géneros literarios), lo que permitirá trabajar desde una corta edad, en función de dichas diferencias, estrategias adecuadas al sexo de cada uno de ellos.

Palabras clave: motivación, lectura, preferencia, género literario, sexo.

\section{ABSTRACT}

In order to develop the reading process, a disposition that allows the reader to relate better to the text is needed. Being motivation an important aspect we can pose the following questions: Who are more motivated: boys or girls? Why? What literary genre do they prefer to read according to their gender?

The present investigation involved 400 children from Initial Education age 5 and 1st and 2nd grade of Primary from schools with different types of management. Two questionnaires were applied: one on reading motivation and another one on literary genres (contact and preference).

The results show differences between boys and girls on their motivation and literary genre preferences, which will make it possible to work on strategies suitable for each gender from an early age, taking those disparities into account.

Key words: motivation, reading, preference, literary genres, gender. 


\section{INTRODUCCIÓN}

Al revisar los resultados obtenidos en comprensión lectora por diversos países en los últimos años se observa un ligero avance, aunque se mantienen los bajos índices, lo que exige plantear estrategias que apunten al mejoramiento de dicha situación. Los profesores juegan un rol principal, ya que después de la familia, son los agentes más cercanos en la formación lectora de niños y niñas. Dicha formación debe hacerse en función de las características del lector, entre ellas su sexo, variable importante en el desarrollo de su personalidad.

\section{PROBLEMA}

El bajo nivel de comprensión lectora asociado con la poca motivación hacia la lectura es preocupante; por ello, los docentes debemos asumir el reto de mejorar las estrategias empleadas en el acercamiento a la literatura infantil.

Los estudios realizados al respecto señalan que la motivación hacia la lectura decae a lo largo de la vida escolar. Conocer esta trayectoria desde los primeros años de la escolaridad, resulta de gran valor para generar estrategias que permitan consolidar los hábitos lectores que impactarán positivamente en la vida académica de los alumnos (Korkeamäki, Tafa, Melo, Orellana, y Gambrell, 2014).

En su trayectoria escolar, los alumnos toman conciencia de tener que leer mucho y según hayan logrado un involucramiento inicial o no, su proceso lector resultará algo gratificante o todo lo contrario (Baldwin, 2012).

No desarrollar una buena motivación hacia la lectura puede llevar incluso al fracaso escolar. Se ha estudiado la relación entre ambas variables, asociándolas con el tratamiento adecuado según el sexo de los alumnos para desarrollar dicha motivación.

$\mathrm{Al}$ respecto, los investigadores denotan que los profesores han tomado conciencia de la importancia de elaborar y desarrollar estrategias diferenciadas para niños y niñas, las cuales les permitan acceder a la lectura con una mayor motivación. Pero, aunque entienden y aceptan que sus 
alumnos por ser varones o mujeres necesitan estrategias diferenciadas para desarrollar mejor sus aprendizajes, en la práctica emplean la misma metodología para ambos sexos, lo cual genera que sólo los niños, o sólo las niñas, se vean más favorecidos o ninguno de ellos (Martino, 2012).

\section{MARCO REFERENCIAL}

\subsection{Motivación a la lectura}

Según Marinak y Gambrell (2010, p. 129): «Los educadores están de acuerdo en que la motivación juega un rol central en el desarrollo de la lectura». Esto implicaría que, sin ésta, el proceso lector se convertiría en algo mecánico y difícil de realizar. Además, los autores afirman: «Mientras que la conciencia fonológica, fonética, vocabulario, fluidez y comprensión permiten a los estudiantes ser lectores diestros y estratégicos, sin la motivación intrínseca para leer, los estudiantes nunca pueden alcanzar todo su potencial como alumnos de alfabetización». Estas afirmaciones nos motivan a encontrar formas efectivas de desarrollar dicha motivación; no se trata de un elemento secundario de la lectura, sino de un aspecto fundamental en su desarrollo que la compromete, pues afecta la capacidad misma del sujeto.

Diversos autores señalan que «la motivación por la lectura es un fenómeno multidimensional y complejo» (Wigfield, 1997; Kirsch, 2002 citados en Orellana, 2012, p. 87). Estudiar dicho fenómeno exige delimitar los aspectos que serán objeto de la investigación. Uno de los más relevantes es el sexo y / o género ${ }^{1}$ como factor que influye en los niveles de motivación.

«Estudios realizados durante la última década apoyan claramente que el género es una variable de gran alcance asociado con la motivación de alfabetización» (Kush y Watkins, 1996; Merisuo-Storm, 2006; Twist, Gnaldi, y Schagen, 2004 citados en Marinak y Gambrell, 2010, p. 130). Dichos estudios nos orientan a investigar, en lectores noveles, el

1 En esta investigación se utiliza el término sexo y está en relación con el término género para hacer referencia a la existencia de dos modalidades de ser humano: ser varón y ser mujer. 
nivel de motivación asociándolo a su sexo para determinar si uno de ellos (femenino o masculino) es el que desarrolla mejores o más altos índices de motivación.

Hace dos décadas ya se tenía una intuición sobre la influencia del sexo sobre el acercamiento hacia la lectura, la cual se corroboraba con resultados académicos. Es así que «las chicas eran las que leían [...] como si se tratara de una clase más intelectual que los chicos» (Camps, 2012, p. 33). Sin embargo faltaba una base científica, la cual hoy se tiene, sobre el porqué de estas conductas evidentes. Se sabe por ejemplo que: «El desarrollo cognitivo del varón es más lento en ciertos tramos de edad en relación, sobre todo, con las habilidades lingüísticas y destrezas verbales» (Calvo, 2009, p. 39). Es decir, existe una evidencia sobre el dominio más avanzado de las niñas sobre el lenguaje, el cual se debe a que el hemisferio izquierdo (que desarrolla esta habilidad) madura mucho antes en la mujer que en el varón (Calvo, 2012), permitiéndole a ésta tener mayor destreza en el uso de sus capacidades lingüísticas pero sólo en el momento que tienen la misma edad que el varón. El que en un período de tiempo la mujer tenga mayor predisposición natural a evidenciar mayor dominio en el aspecto del lenguaje no implica que el varón no pueda llegar a tenerla. Simplemente hay que enfatizar que necesitaría más tiempo para desarrollar dichas capacidades. Por ello es grande la importancia, en la formación inicial de docentes, el que puedan conocer las diferencias entre varones y mujeres en el aprender.

\subsection{GÉNEROS LITERARIOS Y PREFERENCIA SEGÚN EL SEXO DE LOS NIÑOS}

En el estudio de las preferencias literarias asociadas al sexo de los alumnos se tiene en cuenta el tipo de educación que reciben. Se sabe que quienes apoyan la coeducación afirman reiteradamente que en la instrucción mixta los rendimientos de las chicas mejoran, sobre todo en campos de las opciones tradicionales de los chicos (Martial, 2005); sin embargo, dichas opciones no son necesariamente las que las niñas van a preferir, por ejemplo en la literatura infantil no se trata de hacer que todos prefieran tal o cual género sino que se acerquen primero a los que se relacionan más con sus características, necesidades e intereses, y 
progresivamente, por esa motivación inicial, interactúen con los demás. Quizá la ventaja principal de la escuela mixta en este punto es que el aprendizaje social en entornos coeducativos moldea los papeles de género en forma mutuamente complementaria. Pero tal complementariedad a menudo se torna competencia entre sexos (Barrio, 2005) y esto no es un objetivo educativo, al contrario: es una tendencia que se basa en la necesidad de establecer una igualdad entre ambos sexos, olvidando que son sus diferencias las que hacen evidente su singularidad e importancia. Lo dicho da pie a un tratamiento diferencial en cuanto a las estrategias desarrolladas en la escuela ya que no se trata de enseñar convenciones o definir roles, sino de aprovechar las naturales diferencias en la forma de procesar la información para incrementar las posibilidades del aprendizaje.

Por ejemplo, investigaciones como la de Martial (2005, p. 69) señalan que: «Parece que las chicas pueden mantener un espacio personal en escuelas diferenciadas, sobre todo en cuanto a las motivaciones de aprendizaje en materias específicas». Esto lleva a optar por escuelas donde se respete dicho espacio para un mejor desarrollo de la personalidad femenina y una mejor motivación, en este caso hacia la lectura. Académicamente también existen referencias a favor de las escuelas diferenciadas ya que se ha encontrado que: «En relación con las chicas existen estudios que demuestran que sus notas en colegios diferenciados llegan a ser hasta un tercio más elevadas que las de chicas similares en colegios mixtos» (National Foundation fr Educational Research, 2002 citado en Calvo, 2005, p. 280). Con respecto a los varones, destaca en los dos primeros años de primaria su desempeño en sistemas diferenciados (Barquero, 2012). De allí también la importancia de investigar la motivación que tengan tanto niñas como niños en estas edades, relacionándola con el tipo de educación que reciben.

\section{Características de niños y niñas de nivel inicial y primaria}

Con base en distintos estudios se tiene claro que no se trata de una mayor o menor capacidad intelectual de los niños o las niñas lo que los hace diferentes, sino la forma en que desarrollan sus aprendizajes. Calvo (2012, p. 69) al respecto manifiesta que: 
[...], se ha demostrado sobradamente que porcentualmente los varones y las mujeres tienen el mismo promedio de inteligencia aunque las estrategias utilizadas para conseguir un rendimiento parecido sean enormemente diferentes. Nuestros distintos sistemas cerebrales son en su mayoría compatibles y afines pero realizan y cumplen los mismos objetivos y tareas utilizando circuitos distintos.

Con respecto al estudio de la lectura, se encuentra un evidente adelanto en los logros femeninos. Así, otros autores sostienen que:

El desarrollo de la capacidad verbal parece que se consuma en las chicas desde la primera infancia en concordancia con el desarrollo de la inteligencia [...] la superioridad [...] parece estar sujeta, no obstante, a fluctuaciones [...]. Para la edad preescolar [...] las chicas presentan mayores aptitudes idiomáticas orales. En los cuatro primeros años escolares, esa superioridad femenina retrocede, mientras que resurge claramente a partir de los diez años de edad (Tyler, 1965; Cameron/Livson/ Bayley, 1967; Hutt, 1972; Maccoby/Jacklin, 1974; Merz, 1979 en Martial, 2005, pp. 46-47).

Como se puede inferir no se trata de una habilidad exclusiva de las niñas o negada para los niños sino de una facilidad para desarrollarla, que como queda claro, no es constante ni definitiva, pues puede variar según el período escolar y la influencia de otros factores distintos a los del sexo del estudiante.

Existe un cerebro femenino y otro masculino. Y por ello la planificación y desarrollo de las estrategias del docente deben atender a esas diferencias en todo tipo de escuelas. Sin embargo, el reto es mayor cuando se debe atender a ambos sexos a la vez.

\section{Preferencias literarias}

$\mathrm{Al}$ analizar las preferencias literarias de los niños y las niñas es importante relacionarlas con los géneros de la literatura infantil. Básicamente 
son tres los géneros con los que se tiene contacto en la infancia: el género narrativo, el género lírico y el género dramático; cada uno con sus respectivos subgéneros.

Para los niños es fácil identificar el género narrativo con cuentos, historias, leyendas, etcétera; el género lírico con las poesías, aunque también ingresan aquí las canciones y los juegos; por último el género dramático se identifica con el teatro. De estos géneros y subgéneros los niños suelen preferir, según la temática, aquellos relacionados a sus intereses naturales.

\section{Preferencias literarias de los niños}

Según Calvo (2009), los chicos son más objetivos con la lectura. A ellos les aburren los datos íntimos de los personajes, pero disfrutan con la acción, hechos concretos y sus resultados. La obsesión por la igualdad radical entres sexos ha llevado a alejar a los chicos de la literatura, pues se escogen como obligatorias grandes obras literarias, perfectas para chicas, pero que los chicos ven cursis e intimistas. Los temas favoritos para ellos son: la naturaleza (insectos, volcanes, dinosaurios), acción, género épico, aventura, deporte, con personajes que tenga su vida bajo control. Los libros de acción pueden ayudar a motivar a los niños hacia la lectura, huyen de literatura romántica, de historias de desarrollo interior y sentimientos de los personajes. Estas preferencias son claras en los niños, pero deberían ser tomadas por la escuela para la selección de los textos y posteriormente para el desarrollo de estrategias propicias para el acercamiento a la literatura. Como señala Calvo (2009), se debe optar por nuevas formas de enseñanza en las que se elijan obras adaptadas a los gustos varoniles. Esto no implica reforzar estereotipos, sino acercar al alumno a la literatura de modo que luego pueda disfrutar de la poesía, el juego de los sentimientos, etcétera. Efectivamente, lo anterior debe ser tomado como el punto de arranque para que el niño o la niña, según el caso, se predispongan para la lectura. No implica que ellos o ellas no puedan leer las obras importantes del campo literario porque de hacerlo de esa forma también se crearían estereotipos. 


\section{Posibles estrategias}

Desde la perspectiva de una educación diferenciada, Calvo (2009) nos propone estrategias acordes a las características masculinas de los niños, tanto del nivel Inicial como del nivel Primaria.

- Los niños tienen mayor tendencia al aburrimiento y a la distracción que sus compañeras, por ello necesitan emociones fuertes, que les planteen retos que solucionar; por tanto hay que plantearles situaciones en que ellos sean protagonistas de la acción, por ejemplo a través de los personajes encontrados en sus lecturas.

- Debido a su curiosidad por el funcionamiento de las cosas y de los fenómenos, será fundamental pedirles una explicación sobre los mismos cuando aparezcan en una historia, de forma que a través de este reto, ellos se involucren más con el texto.

- Es necesario planificar estrategias como rondas, representaciones, juegos de acción referidos a los libros que están leyendo, de forma que siempre haya un espacio para canalizar la energía generada por el interés en la lectura y por la naturaleza misma de los niños.

- Trabajar con el ritmo y la sonoridad, acerca a los varones a la literatura a través de la diversión y la acción que podemos encontrar en las canciones y en los juegos infantiles.

- Son fundamentales los modelos de héroes o protagonistas de libros o películas que ayuden a distinguir el comportamiento masculino del agresivo. Se trata de captar la atención de los niños y enfocarla en aspectos positivos de los personajes, aprovechando el interés natural por la acción que éstos desarrollan en las historias.

\section{Preferencias literarias de las niñas}

Las preferencias de las niñas también se orientan según sus características, entre ellas las intelectuales. Según Ahedo (2012, p. 51): «En cuanto a la inteligencia, en ella está más personalizada lo que implica que sea más subjetiva». De aquí podemos inferir que los géneros 
preferidos serán aquellos que permitan desarrollar dicha subjetividad Por eso, el género predilecto será aquel que se relacione con sentimientos, emociones, vivencias íntimas (por ejemplo historias románticas, cuentos de hadas, poesía, canciones tiernas, etcétera). Además se debe recordar la facilidad para establecer relaciones que tienen las niñas, que según un estudio de Courten-Myer (1999 citado en Norfleet, 2015), ha encontrado mayores conexiones entre sus neuronas que entre las de un varón.

Autores como Witelson (1989) y Hines (2004) citados en Norfleet (2015) señalan también que el itsmo del cuerpo calloso del cerebro es más largo en la mujer, lo cual implica que surgen conexiones entre los dos hemosferios cerebrales, por ejemplo en cuanto al desarrollo del lenguaje, mientras que en el varón no se daría así. Esto se complementa con lo encontrado por Halpern (2000) y Kimura (2000), citados en Norfleet (2015, p. 22), quienes sotienen que «cuando la memoria involucra palabras, experiencias o lugares, las mujeres tienen mejor recuerdo que los varones».

\section{Posibles estrategias}

Desde la perspectiva de una educación diferenciada (Norfleet, 2015, pp. 15, 17, 18), Norfleet nos propone estrategias acordes a las características femeninas de los niñas, tanto del nivel Inicial como del nivel Primaria.

- Antes de entrar en los detalles de un tema, empezar por discutir comprensivamente el material. Por ejemplo, hablar de una característica común, en niñas pequeñas deben ser características observables, como formas o colores. Trabajar con convenciones sobre los colores a utilizar para determinadas acciones dentro de las actividades de lectura.

- Para las niñas, la competencia no las activa sino que incluso las bloquea. Ellas necesitan de la socialización, establecer redes sociales protectoras, para lo que es importante la colaboración y cooperación entre compañeras (Calvo, 2009). Por eso se pueden trabajar en 
grupos, lecturas asociadas a temas como las matemáticas o las ciencias para que en equipo encuentren, de forma amena y con seguridad en sus habilidades, el soporte para el desarrollo de estas áreas.

- En tareas de dramatización, pedirles que exageren expresiones de sentimientos. Proporcionarles ejemplos de mensajes con lenguaje corporal, comparando lo que cada una de ellas pueda haber comprendido y hacerles ver la importancia de la expresión como acompañante del lenguaje verbal.

Otras opciones:

- Disfrutar del conocimiento manifestando esa alegría a través de canciones, rimas, elementos melódicos que acompañen la lectura o el trabajo con los textos.

Frente a estas diferencias encontradas entre varones y mujeres, a nivel cerebral y de procesamiento de la información, así como de las preferencias por distintos géneros o subgéneros literarios, se justifica una investigación sobre el tratamiento que se da actualmente a dichas diferencias desembocando en la motivación que tienen niños y niñas en los niveles Inicial y Primaria en todo tipo de escuelas.

Debemos enfatizar que los objetivos, contenidos y las metas deben ser los mismos; sin embargo, los métodos pedagógicos y las estrategias deben ser distintos si aspiramos a la excelencia personal y académica de los niños y las niñas, respetándoles de acuerdo a esas dos modalidades del ser humano. La investigación también aporta en este sentido ya que permite inferir relaciones entre los resultados y las formas de enseñanza usadas en los colegios encuestados, según el nivel alcanzado en cada uno de los test.

\section{EL ESTUDIO}

Esta investigación partió de la hipótesis que las niñas están más motivadas que los niños por la lectura, ya que diversas investigaciones apuntan a que ésta es un área que desarrollan con precocidad con 
respecto a los niños y para la que tienen desplegadas más habilidades en los primeros grados de escolaridad. Además se esperaba que las niñas prefirieran el género lírico, más subjetivo, y los niños el género narrativo, referido más a la acción. Por otro lado es necesario recoger información sobre el contacto que tienen los niños con cada uno de los géneros, la frecuencia de ese contacto, la opción que tengan para tener dicho contacto y si perciben el aspecto lúdico de los géneros que les propicie un acercamiento más efectivo a los mismos.

Se plantearon como objetivos:

- Describir la diferencia en la motivación por la lectura, según el género de los niños.

- Identificar los géneros literarios con los que los niños tienen mayor y mejor contacto.

- Determinar los géneros literarios preferidos por los niños, según su sexo.

La metodología utilizada fue cuantitativa y se aplicaron dos cuestionarios validados bajo la técnica de la encuesta. Uno de ellos fue el Me and My Reading Profile, extraído y traducido de la investigación de Marinak, Malloy, Gambrell, y Mazzoni (2015), y Mis géneros literarios preferidos elaborado por las investigadoras. Cabe destacar que del primer instrumento sólo se aplicó el cuestionario y no la entrevista que lo acompaña, debido a la disponibilidad limitada de interacción con los alumnos en las instituciones encuestadas. Ambos cuestionarios utilizan una escala tipo Lickert para las respuestas. Los sujetos de la investigación fueron niños de Inicial 5 años, y $1^{\circ}$ y $2^{\circ}$ grado de Primaria, ya que en investigaciones de Mckenna et al. (1995), citado en Marinak y Gambrell (2010), encuentran una significativa erosión de la actitud hacia la lectura, ya sea ésta académica o recreativa, alrededor del cuarto grado, por lo que se prefirió trabajar con edades más tempranas para tener un primer acercamiento e incluso posible predicción del comportamiento lector posterior de dichos alumnos, según el nivel de motivación alcanzado. 
La muestra estuvo conformada por 400 niños y niñas de la provincia de Piura-Perú, distribuidos en cuatro colegios de educación separada, tres de mujeres (ESF) y uno de varones (ESM), y cinco colegios mixtos-coeducación (EC).

Para determinar la fiabilidad de ambos test se ha realizado un análisis de consistencia interna a través del Alfa de Cronbach.

Los resultados obtenidos después de realizar el análisis a través del programa IBM SPSS Statistics 22 son los valores que se muestran en la siguiente tabla:

Tabla 1. Análisis de consistencia interna. Alfa de Cronbach

\begin{tabular}{|l|c|c|}
\hline \multicolumn{1}{|c|}{ Test } & Alfa de Cronbach & Nivel de fiabilidad \\
\hline Yo y mi perfil lector & 0.865 & Bueno \\
\hline Géneros literarios & 0.662 & Cuestionable \\
\hline
\end{tabular}

Según la anterior tabla, los test han logrado obtener un alfa mayor a 0.5 lo que indica una garantía de fiabilidad aceptable de los instrumentos aplicados.

\section{RESULTADOS E INTERPRETACIÓN}

Para la elaboración de gráficos y cálculos se emplearon dos herramientas informáticas: Excel de Microsoft Office 2010 y SPSS Statistics 22 de IBM.

Los resultados se muestran en gráficos de barras, presentando los porcentajes alcanzados en cada factor analizado según el sexo. Para comenzar, se muestra la distribución del alumnado por nivel, institución y sexo en el primer cuestionario Yo y mi perfil lector. 
Tabla 2. Cuestionario "Yo y mi perfil lector».

Cantidad de alumnos según nivel, escuela y género

\begin{tabular}{|c|c|c|c|c|}
\hline Nivel & Escuelas & M & $\mathbf{F}$ & Total \\
\hline \multirow{5}{*}{ Inicial - 5 años } & EC1 & 11 & 13 & 24 \\
\hline & ESF1 & 0 & 20 & 20 \\
\hline & EC2 & 11 & 10 & 21 \\
\hline & ESM1 & 26 & 0 & 26 \\
\hline & EC3 & 9 & 2 & 11 \\
\hline \multirow{6}{*}{ Primaria - Primero } & ESF1 & 0 & 27 & 27 \\
\hline & ESF2 & 0 & 20 & 20 \\
\hline & EC1 & 6 & 7 & 13 \\
\hline & EC2 & 19 & 12 & 31 \\
\hline & ESM1 & 25 & 0 & 25 \\
\hline & ESF3 & 0 & 30 & 30 \\
\hline \multirow{6}{*}{ Primaria - Segundo } & ESF1 & 0 & 23 & 23 \\
\hline & ESF2 & 0 & 36 & 36 \\
\hline & EC1 & 4 & 7 & 11 \\
\hline & EC2 & 17 & 13 & 30 \\
\hline & ESM1 & 26 & 0 & 26 \\
\hline & EC3 & 10 & 16 & 26 \\
\hline \multicolumn{2}{|l|}{ Total } & 164 & 236 & 400 \\
\hline \multicolumn{2}{|l|}{$\%$} & $34 \%$ & $66 \%$ & $100 \%$ \\
\hline
\end{tabular}


Según la anterior tabla, la población mayor en el primer cuestionario (152 estudiantes) está en segundo grado, de la cual un gran porcentaje son niñas (92). En primer grado, de 146 alumnos, 96 son niñas, y en Inicial 5 años de los 102 estudiantes, sólo 45 son niñas. Como se observa, la diferencia entre niños y niñas es algo considerable, pues se trata de $18 \%$ de diferencia a favor de las niñas.

\section{Motivación hacia la lectura}

Los resultados obtenidos se presentan en la siguiente figura:

Figura 1. Cuestionario «Yo y mi perfil lector». Medias totales

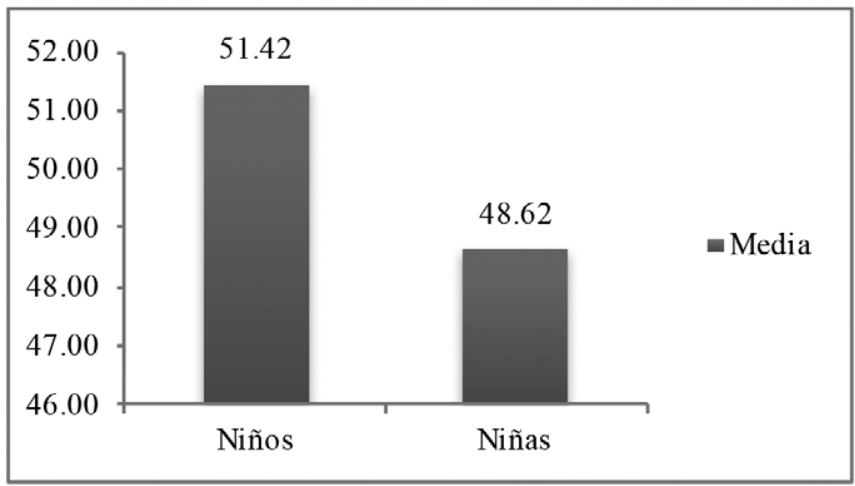

La anterior figura representa gráficamente los resultados de las medias totales del cuestionario Yo y mi perfil lector. Como se observa, la diferencia a favor de los niños es de 2.8 puntos con lo cual, aunque en forma limitada, la hipótesis de la que partimos — referida a una mejor motivación en las niñas- no se cumple en la población encuestada a pesar que son más niñas que niños. Así mismo se aprecia que el nivel de motivación hacia la lectura en general es bastante bueno, pues alcanza más de 48 de los 60 puntos del cuestionario. 


\section{Preferencia por los géneros literarios}

El segundo cuestionario analiza los géneros literarios con que los alumnos tienen mayor y mejor contacto, así como la preferencia por alguno de ellos, según el sexo de los estudiantes.

A continuación se muestra la distribución del alumnado por nivel, institución y sexo en el segundo cuestionario "Mis géneros literarios preferidos".

Tabla 3. Cuestionario "Mis géneros literarios preferidos".

Cantidad de alumnos según nivel, escuela y género

\begin{tabular}{|l|l|c|c|c|}
\hline \multicolumn{1}{|c|}{ Nivel } & Escuelas & M & F & Total \\
\hline \multirow{4}{*}{ Inicial - 5 años } & EC1 & 11 & 13 & 24 \\
\cline { 2 - 5 } & ESF1 & 0 & 20 & 20 \\
\cline { 2 - 5 } & EC2 & 11 & 13 & 24 \\
\cline { 2 - 5 } & ESM1 & 26 & 0 & 26 \\
\cline { 2 - 5 } & EC3 & 8 & 3 & 11 \\
\hline \multirow{5}{*}{ Primaria - } & ESF1 & 0 & 30 & 30 \\
\cline { 2 - 5 } & ESF2 & 0 & 20 & 20 \\
\cline { 2 - 5 } & EC1 & 6 & 7 & 13 \\
\cline { 2 - 5 } & EC2 & 20 & 12 & 32 \\
\cline { 2 - 5 } & ESM1 & 26 & 0 & 26 \\
\cline { 2 - 5 } & ESF3 & 0 & 30 & 30 \\
\hline \multirow{5}{*}{$\begin{array}{l}\text { Segimaria - } \\
\text { Segundo }\end{array}$} & ESF1 & 0 & 22 & 22 \\
\cline { 2 - 5 } & ESF2 & 0 & 27 & 27 \\
\cline { 2 - 5 } & EC1 & 4 & 5 & 9 \\
\cline { 2 - 5 } & EC2 & 16 & 14 & 30 \\
\cline { 2 - 5 } & ESM1 & 26 & 0 & 26 \\
\cline { 2 - 5 } & EC3 & 10 & 16 & 26 \\
\hline Total & & $\mathbf{1 6 4}$ & $\mathbf{2 3 2}$ & $\mathbf{3 9 6}$ \\
\hline \multirow{3}{*}{} & & & \\
\hline
\end{tabular}




\section{Contacto con el género}

En este aspecto es fundamental que los niños tengan contacto con todos los géneros; sin embargo, los resultados muestran que no es así.

Figura 2. Cuestionario «Mis géneros literarios preferidos». Porcentaje de mayor contacto con el género

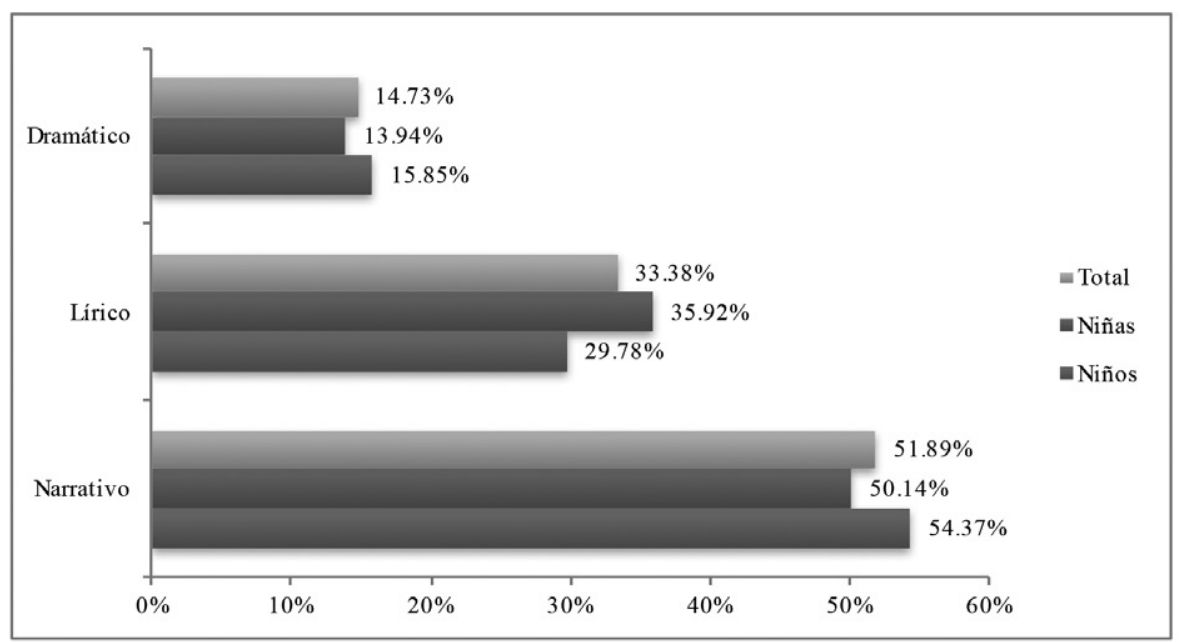

En la figura anterior se muestra que, en general, es el género narrativo con el que se tiene mayor contacto. Así mismo se observa que son los niños quienes tienen casi 3\% más de contacto con este género, en comparación con las niñas. Con respecto al género lírico, el contacto es mayor de las niñas con él, ya que tienen casi $4 \%$ de diferencia con respecto a los niños. El género dramático es con el que ambos sexos tienen menos contacto.

En cuanto a la calidad de ese contacto, podemos analizarlo en términos de frecuencia, opción y la dimensión lúdica que los niños aprecien en cada género literario. 


\section{Frecuencia}

Es importante propiciar, tanto en la vida familiar como en la escuela, momentos para la lectura espontánea y frecuente. Así, tanto en el nivel Inicial como en Primaria, la frecuencia con la que el niño toma contacto con un determinado género puede influir en la preferencia por éste y también en su motivación hacia la lectura.

Figura 3. Cuestionario «Mis géneros literarios preferidos». Porcentaje de frecuencia de contacto con el género

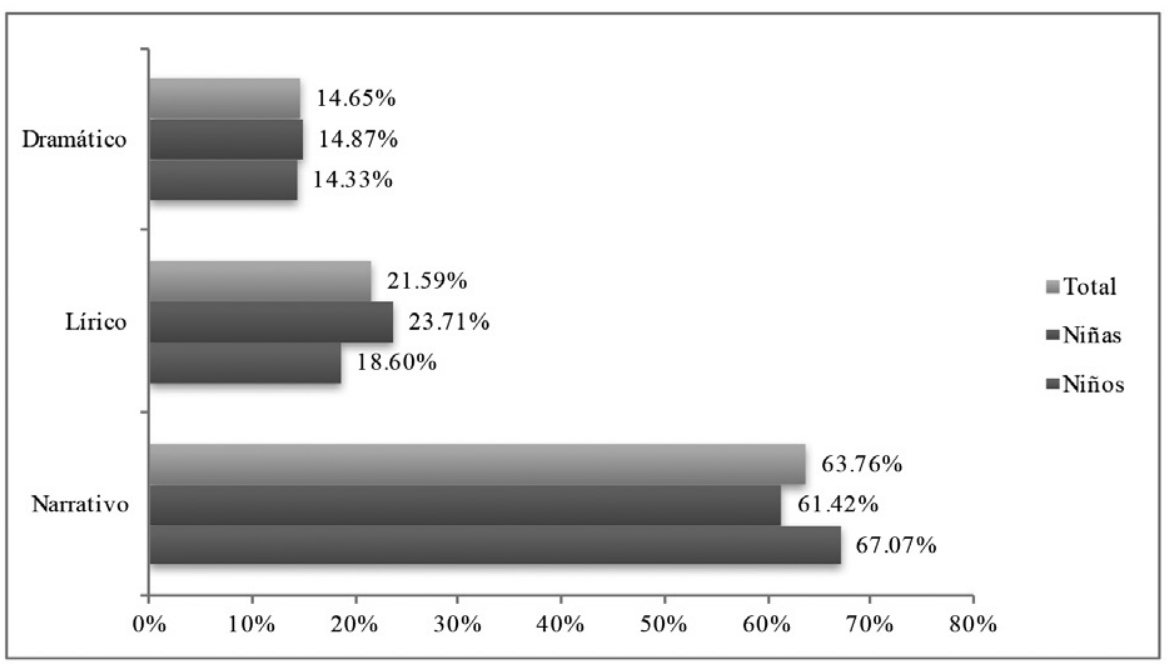

Según esta figura se observa que es el género narrativo aquél con el que los niños toman contacto con más frecuencia. Es decir se relacionan más con literatura referida a historias, acción, personajes de todo tipo, etcétera. De acuerdo a la temática del libro, se generará mayor preferencia en los niños o las niñas y, por lo tanto, una mayor motivación hacia la lectura. 


\section{Opción}

Es necesario que los libros estén al alcance de los niños, generando las mismas posibilidades para optar por los de uno u otro género. Es decir, contar con una biblioteca variada y accesible. Aunque esto es importante desde el nivel Inicial, no deja de serlo en el nivel Primaria, porque favorece el espectro de elección del alumno y puede aumentar su motivación hacia la lectura por la posibilidad de encontrar nuevas experiencias en los distintos géneros.

Figura 4. Cuestionario «Mis géneros literarios preferidos». Porcentaje según opción para el contacto con el género

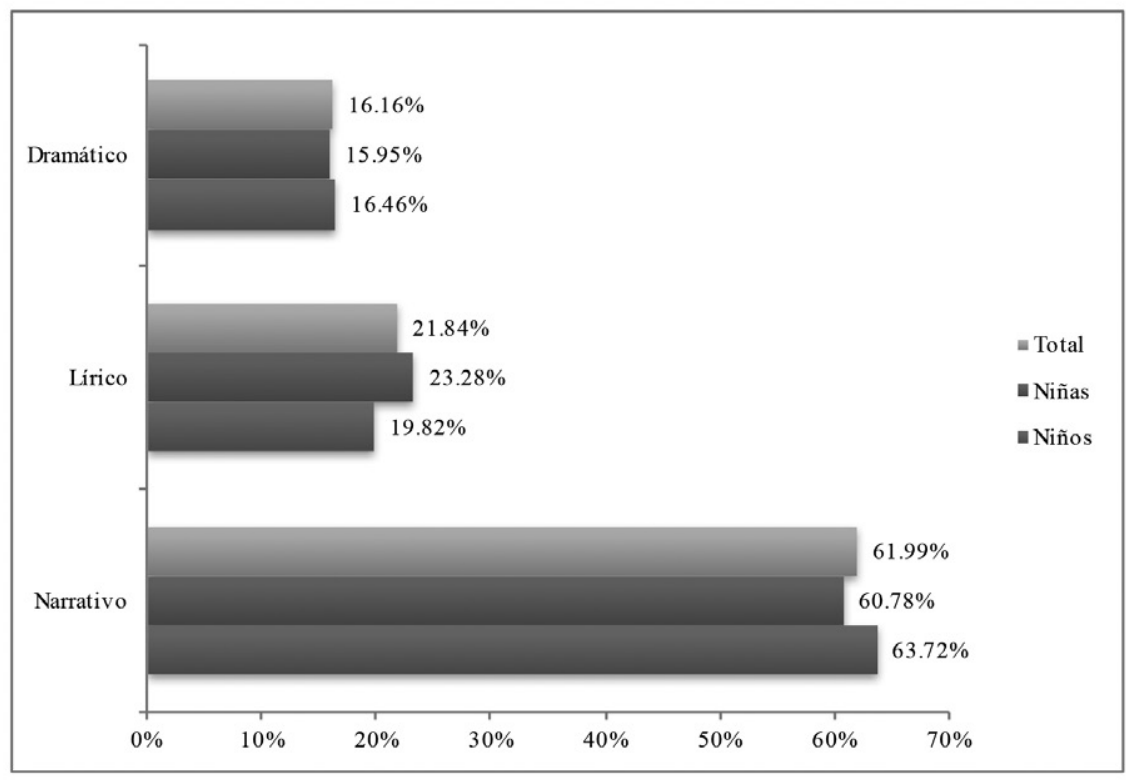

Según la figura anterior, el género narrativo es el que tiene más opción de ser contactado por los alumnos. Es decir, es mucho más probable encontrar libros de cuentos e historias en casa o en el colegio, que libros de poesía, juegos o de teatro. 
Además es importante la manera en que disponemos de los libros para propiciar el contacto de los alumnos con ellos, de esa forma los niños tanto de Inicial como de Primaria al tener la posibilidad de interactuar con los textos aprenden a cuidarlos.

\section{Dimensión lúdica}

El juego del lenguaje o de las palabras puede apreciarse mejor en un género que en otro. Normalmente lo asociamos más al género lírico pues en él encontramos los juegos, las rimas, las poesías y las canciones.

Figura 5. Cuestionario "Mis géneros literarios preferidos". Porcentaje según la dimensión lúdica que se aprecia en el género

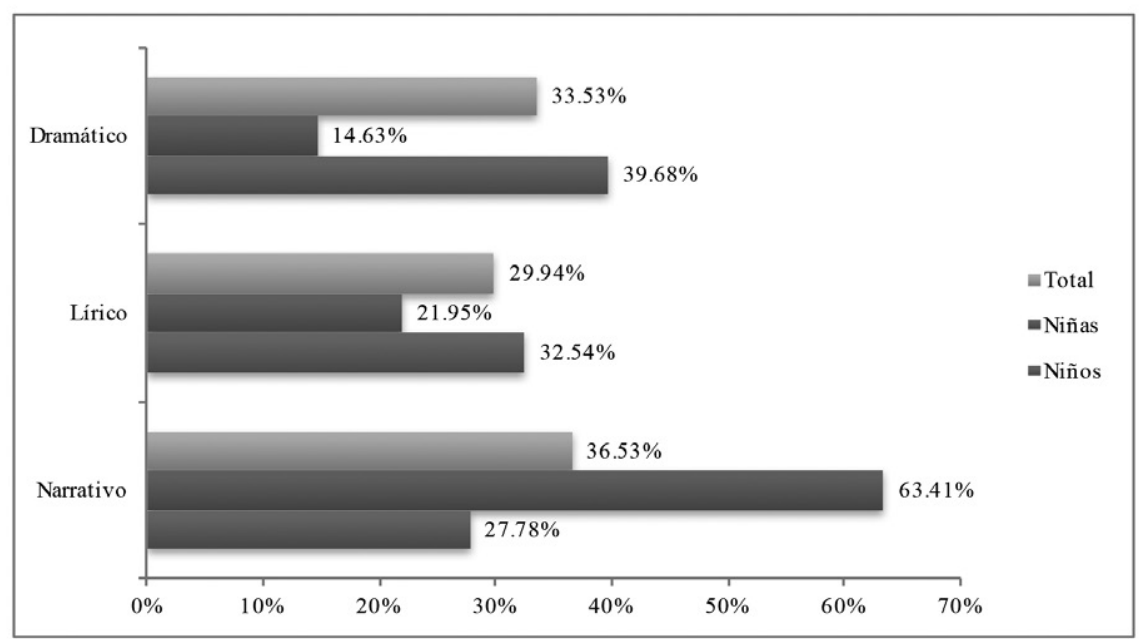

En los resultados mostrados en la figura 5 se observa que las niñas encuentran más lúdico el género narrativo y los niños el género dramático. En el caso de los últimos se entiende el resultado, pues dentro de las características de los niños está la predisposición a la acción, la cual 
es propia del género dramático, ya que implica ejecutar acciones de los personajes reproduciendo una historia.

En el caso de las niñas es probable que el «juego» sea de tipo menos concreto y se refiera a la posibilidad de vivir las historias y recrearse imaginándolas cuando las leen o escuchan.

\section{Preferencia por el género literario}

La preferencia por el género no sólo se asocia al género en sí, si no a los subgéneros y a las temáticas recreadas en las obras literarias.

Figura 6. Cuestionario "Mis géneros literarios preferidos". Porcentaje de preferencia por uno de los géneros

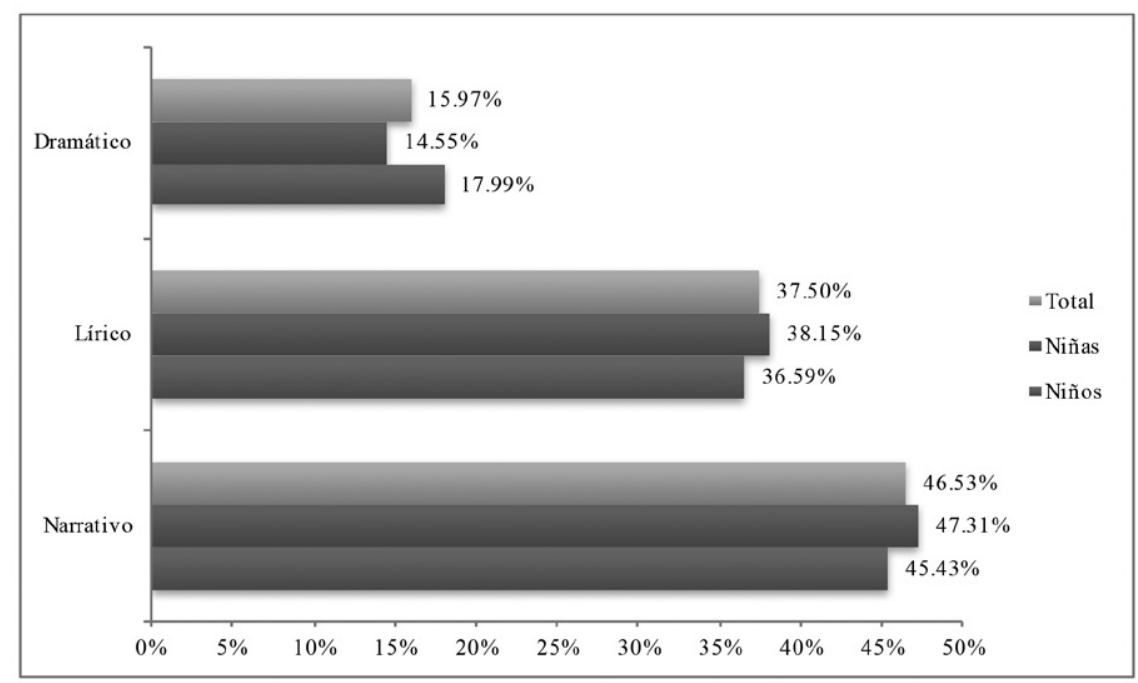

En la figura 6 se señala que el género preferido por los niños es el narrativo, con lo cual la hipótesis planteada quedaría probada parcialmente, pues el género preferido por las niñas no es el lírico, sino 
también el narrativo. En estas preferencias, como se ha mencionado en el marco referencial, influye la temática abordada en la obra literaria.

Figuras 7 y 8. Cuestionario "Mis géneros literarios preferidos».

Porcentaje de preferencia por uno de los géneros en Inicial 5 años y Primer grado de Primaria respectivamente
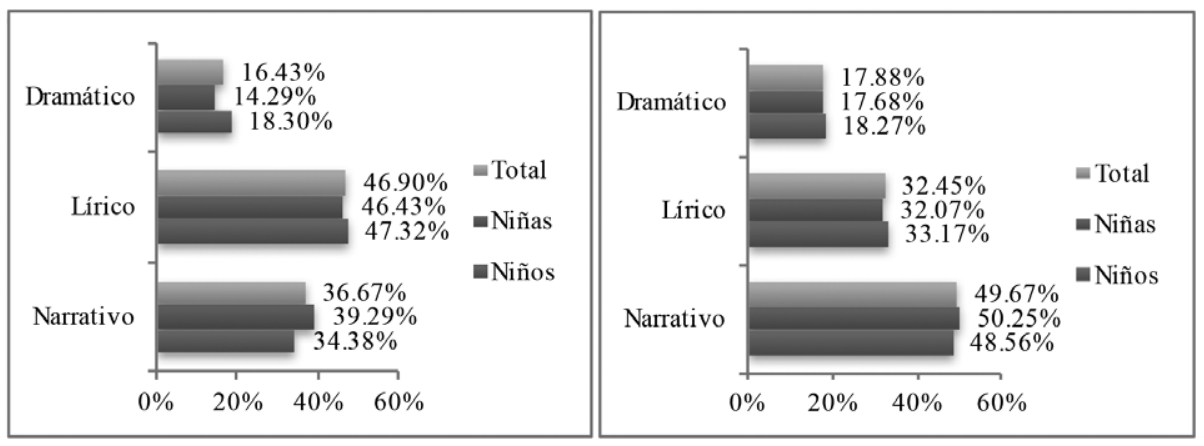

Figura 9. Cuestionario "Mis géneros literarios preferidos».

Porcentaje de preferencia por uno de los géneros en

Segundo grado de Primaria

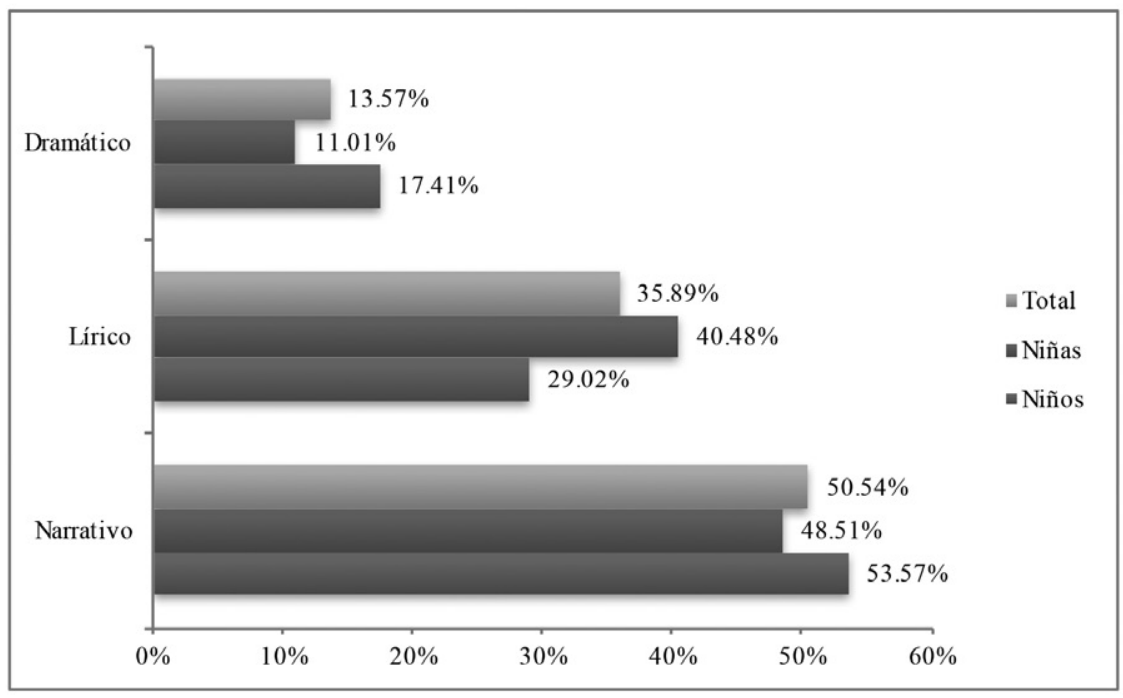


Al observar y comparar las figuras 7, 8 y 9, observamos que es en Inicial 5 años donde niños y niñas, en un $46 \%$ más, prefieren el género lírico. Sin embargo, es en primer y segundo grado de Primaria donde se da la mayor preferencia por el género narrativo en ambos sexos. Esta diferencia puede relacionarse con el tratamiento más lúdico del género lírico en Inicial que en Primaria, y también con la preferencia del género narrativo en las estrategias didácticas utilizadas por los docentes en los momentos de lectura.

\section{DISCUSIÓN}

Los resultados encontrados en la población estudiada coinciden con los de Barquero (2012), quien encontró que: al utilizar estrategias didácticas acordes con las características de los varones, puede motivárseles para que se involucren en la literatura infantil, mejorando su autoconcepto lector y valorando la lectura en su vida.

Las mujeres obtienen los resultados más bajos en relación a los cuestionarios (tipo test). Esto puede deberse, tal como lo explica Calvo (2009), a que las mujeres — siendo más inductivas que los varonesnecesitan recopilar detalles para llegar a la regla general. Así, el uso de alternativas en los cuestionarios limita su capacidad para relacionarse con el tema, pues ellas requieren conversar sobre el mismo.

Otra explicación para estos resultados es que el cerebro en ambos sexos es altamente plástico y que, con las estrategias adecuadas, puede mejorarse una capacidad que inicialmente era precaria, por ejemplo el pensamiento lógico matemático en las mujeres o las destrezas verbales en los varones (Calvo, 2012).

Esto explica que la hipótesis inicial no se confirme, pero favorezca en este caso a los varones pues han logrado buenos niveles de motivación hacia la lectura en Inicial 5 años, y Primer y Segundo grado de Primaria. 
En cuanto a la preferencia por los géneros literarios tampoco sorprenden los resultados, pues el tratamiento didáctico que se da a éstos en ambos niveles -Inicial y Primaria-, va reforzando ese acercamiento y preferencia por los mismos.

\section{CONCLUSIONES Y RECOMENDACIONES}

- Los niños de Inicial 5 años, y de Primer y Segundo grado de Primaria tienen mejor nivel de motivación que las niñas.

- En cuanto al contacto con los géneros, el favorecido es el género narrativo, lo cual explica también que sea el favorito de ambos sexos. Tanto por frecuencia como por opción y por dimensión lúdica, aunque en Inicial 5 años es superado en este aspecto por el género lírico, el género narrativo es aquel al que tienen más acceso los niños y las niñas, ya sea por intervención de un adulto (lectura en voz alta) o porque ellos eligen y pueden leerlo.

- Como recomendación puede señalarse que es importante generar espacios en las escuelas para desarrollar estrategias acordes con las características de los niños, pues los resultados señalan que aunque neurocognitivamente no es un área de fortaleza en los varones en las primeras edades de escolaridad, con trabajo adecuado, los chicos logran rendir igual o mejor que las niñas, quienes por naturaleza desarrollarían con más facilidad el área de lectura.

- También es importante que las escuelas cuenten con variedad de textos de literatura infantil, con temática adecuada a los intereses de varones y mujeres de forma que la preferencia por algún género literario no se vea influenciada sólo por la posibilidad de acceso a él. 


\section{REFERENCIAS}

Ahedo, J. (2012). Fundamento antropológico de la separación escolar de chicos y chicas. En E. V. (comp.), Nuevo paradigma escolar. Educación single-sex o diferenciada (págs. 39-60). Rosario: PROMESA-Ediciones Logos-PROCODES.

Baldwin, P. Y. (2012). Motivación por la lectura: Aproximaciones a las diferencias entre niños y niñas. En E. Vierheller, Nuevo paradigma escolar. Educación single-sex o diferenciada (págs. 85-111). Rosario: PROMESA-Ediciones Logos-PROCODES.

Barquero, A. (2012). Diferencias estructurales, hormonales y funcionales que inciden en el aprendizaje entre varones y mujeres. En E. Vierheller, Nuevo paradigma escolar. Educación single-sex o diferenciada (págs. 113-135). Rosario: PROMESA-Ediciones Logos-PROCODES.

Barrio, J. (2005). Educación diferenciada, una decisión razonable. Pamplona: EUNSA.

Calvo, M. (2005). Todos iguales pero diferentes. El derecho a una educación diferenciada. En J. B. (Ed.), Educación diferenciada, una opción razonable (págs. 263-298). Pamplona: EUNSA.

Calvo, M. (2009). Guía para una educación diferenciada. España: Toro Mítico.

Calvo, M. (2012). En busca del equilibrio entre natruraleza y cultura. En E. Vierheller, Nuevo paradigma ecolar. Educación single-sex $o$ diferenciada (págs. 61-81). Rosario: PROMESA-Ediciones Logos-PROCODES.

Camps, J. (2012). La escuela diferenciada por sexos y la cultura escolar. En E. V. (comp.), Nuevo paradigma escolar. Educación single sex o diferenciada (págs. 17-38). Rosario: PROMESA-Ediciones Logos-PROCODES. 
Korkeamäki, R., Tafa, E., Melo, C., Orellana, P., y Gambrell, L. (21 y 22 de Agosto de 2014). Motivación Lectora en niños y niñas que se inician en la lectura: una aproximación intercultural. Obtenido de Web del Tercer Congreso Interdisciplinario de Investigación en Educación.: http:/ / www.ciie2014.cl/download.php?file=sesiones / 42.pdf

Marinak, B., \& Gambrell, L. (2010). Reading Motivation: Exploring the Elementary Gender Gap. Literacy Research and Instruction, 129-141.

Marinak, B., Malloy, J., Gambrell, L., \& Mazzoni, S. (2015). Me and my Reading Profile. A Tool for Assesing Early Reading Motivation. The Reading Teacher, 51-62.

Martial, I. V. (2005). Coeducación y educación separada. En J. B. (Ed.), Educación diferenciada, una opción razonable (págs. 21-87). Pamplona: EUNSA.

Martino, M. (2012). La influencia del sexo en el proceso de enseñanza-aprendizaje del inglés como lengua extranjera. En E. V. (comp.), Nuevo paradigama escolar. Educación single-sex $o$ diferenciada (págs. 137-184). Rosario: PROMESA-Ediciones Logos-PROCODES.

Norfleet, A. (Setiembre de 2015). New Perpectives on Teaching Boys anf Girls. Obtenido de http://www.educacion.udep.edu.pe/ juga/diplomado/CAP_2_MALE.pdf

Norfleet, A. (Setiembre de 2015). New Perpectives on Teaching Boys anf Girls. Obtenido de http: / / www.educacion.udep.edu. pe/juga/diplomado/CAP_1.pdf

Orellana, P. B. (2012). Motivación por la lectura: Aproximaciones a las diferencias entre niños y niñas. En E. Vierheller, Nuevo paradigma escolar. Educación single-sex o diferenciada (págs. 85-111). Rosario: PROMESA-Ediciones Logos-PROCODES. 